\title{
ON THE ORDER OF LINEAR HOMOGENEOUS GROUPS *
}

\author{
(SUPPLEMENT)
}

BY

\section{H. F. BLICHFELDT}

$\S 1$. The author has given a superior limit to the value of a prime $p$ which may divide the order of a finite, primitive group of linear homogeneous substitutions, of determinants unity, in $n$ variables, namely $p \leqq(n-1)(2 n+1)$ (with reductions for the cases $n \leqq 6) . \dagger \mathrm{He}$ has also proved that, if such a group $G$ contains a substitution $S$ of variety $m(\leqq n)$ and of order $p^{a+c} \geqq m p^{c}$, then will $G$ contain an invariant subgroup $H$ (or be itself such a group), which possesses the property that, if $V$ be a substitution of $G$, and $T$ one of $H$, then is $(V)_{p} \equiv(V T)_{p}(\bmod p)$.

The groups not containing $H$ being by far the most difficult to determine, at least when $n$ is small, the author proves, in the present paper, a theorem supplementing the one just stated, giving a lower general limit to the highest power of $p$ which may divide the order of a group $G$ not containing $H$, than flows from the theorem stated. The paper also gives further reductions to the general formula for the limit to $p$, as well as to the special cases when $n \leqq 6$.

§2. Theorem 14. If a group $G$ in $n$ variables has an abelian subgroup $K$ of order $p^{a} \geqq p^{n}$, then will $G$ have an invariant subgroup $H$ containing a subgroup of $K$ of order $p^{a-n+1}$. If $S$ is any substitution of $H$, and $V$ any of $G$, then is $(V)_{p} \equiv\left(V S^{r}\right)_{,}(\bmod p)$.

Let $K$ be written in canonical form, and let its substitutions be $I=S_{0}, S_{i}$ $\left(i=1,2, \cdots, p^{n}-1\right)$, with the multipliers

$$
(1,1,1, \ldots, 1),\left(\theta_{1, i}, \theta_{1, i} \theta_{2, i}, \theta_{1, i} \theta_{3, i}, \cdots, \theta_{1, i} \theta_{n, i}\right) .
$$

To the determinant (6) of $L$ - $G$ II will correspond the following matrix of $p^{a}$ rows and $1+m$ columns, where $m$ corresponds to "variety" of theorem 10 :

* Presented to the Society (Chicago), April 14, 1906. Received for publication February 13, 1906.

† On the Order of Linear Homogeneous Groups, Transactions, vol. 4 (1903), pp. 387-397.

$\ddagger$ On the Order of Linear Homogeneous Groups (second paper), ibid., vol. 5 (1904), pp. 310325. The theorem, stated on page 315 of the paper, is numbered 10 . These two articles will be referred to by $L \cdot G$ I and $L-G$ II, respectively. 


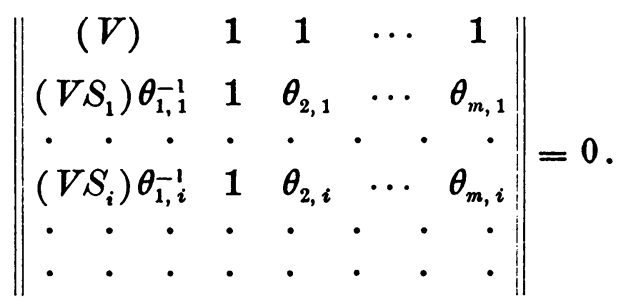

Now, to this matrix * may be added $p^{a-m+1}$ rows of the form

$$
\left(V S_{i}\right)-(V)+(1-e) X_{i}, 0,0, \ldots, 0,
$$

where $\theta$ is a root of $\rho^{p a}-1=0$, and where $X_{i}$ is a linear function of the quantities $(V),\left(V S_{1}\right), \cdots$, with coefficients that are integral functions of $\theta$, the numerical coefficients entering being integers or fractions whose denominators are prime to $p$. To prove this proposition we shall assume it true for all possible matrices of similar form, but containing fewer columns. Under this assumption, we may add to the matrix (1) $p^{a-m+2}-1$ rows of the form

$$
\left(V S_{i}\right)-(V)+(1-\theta) X_{i}, 0,0, \ldots, 0, Y_{i},
$$

where the quantities $Y_{i}$ are integral functions of $\theta$ with numerical coefficients which are integers or fractions whose denominators are prime to $p$. Let us suppose that $i$ can take any one of the values $2,3, \cdots, p^{a-m+2}$.

It is readily proved that $Y_{i}$ may be written in the form

$$
Y_{i}=a_{i}(1-\theta)^{a_{i}}+b_{i}(1-\theta)^{\beta_{i}}+c_{i}(1-\theta)^{\gamma_{i}}+\cdots \quad\left(a_{i}<\beta_{i}<\gamma_{i}<\cdots\right),
$$

where $a_{i}$ is one of the numbers $1,2, \ldots, p-1$, while $b_{i}, c_{i}, \ldots$ are integers or fractions whose denominators are prime to $p$. We shall suppose the rows arranged in such a way that

$$
\alpha_{i}=\alpha_{3}=\cdots=\alpha_{k+1}<\alpha_{j} \quad\left(j=k+2, k+3, \cdots, p^{a-m+2}\right) .
$$

First, if $k \leqq(p-1) p^{a-m+1}$, we obtain $p^{a-m+2}-(k+1) \geqq p^{a-m+1}-1$ rows of the form

$$
\left(V S_{j}\right)-(V)+(1-\theta) X_{j}-\frac{Y_{j}}{Y_{2}}\left[\left(V S_{2}\right)-(V)+(1-\theta) X_{2}\right], 0,0, \ldots, 0 ;
$$

1. e., of the form

$$
\left(V S_{j}\right)-(V)+(1-\theta) X_{j}^{\prime}, 0,0, \ldots, 0
$$

Here the quantities $X_{j}^{\prime}$ are easily proved to be of the same general form as the quantities $X_{i}$, and hence the proposition assumed true for a matrix with $m$ columns, is proved true for one of $m+1$ columns.

Next, it $c>(p-1) p^{a-m+1}$, we proceed thus: The numbers $a_{2}, a_{3}, \cdots, a_{k+1}$, taking the values $1,2, \cdots, p-1$ only, may be separated into lots, among

\footnotetext{
* It is to be noticed that no two of the last $m$ columns are identical.
} 
which there must be one of $l \geqq k /(p-1) \geqq p^{a-m+1}$ numbers (say $\left.a_{2}, a_{3}, \ldots a_{l+1}\right)$, having one and the same value $a_{2}$. Then we derive $l-1 \geqq p^{a_{-m+1}}-1$ rows of the form

$\left(V S_{i}\right)-(V)+(1-\theta) X_{i}-\frac{Y_{1}}{Y_{2}}\left[\left(V S_{2}\right)-(V)+(1-\theta) X_{2}\right], 0,0, \ldots, 0 ;$

i. e., of the form

$$
\left(V S_{i}\right)-\left(V S_{2}\right)+(1-\theta) X_{i}^{\prime}, 0,0, \ldots, 0 .
$$

But, if $l-1$ such rows result, it is readily proved that we could obtain $l-1$ rows of the form

$$
\left(V S_{2}^{-1} S_{i}\right)-(V)+(1-\theta) X_{i}^{\prime \prime}, 0,0, \cdots, 0,
$$

and the proposition is fully proved.

$\S 3$. Let us now consider the matrix (1). We may add the $p^{a-m+1}-1$ rows (2) to the $p^{a}$ rows in (1). Provided that at least one of the determinants of $m^{2}$ elements, contained in the matrix obtained by erasing the first column of (1), does not vanish, we get, then, $p^{a-m+1}$ equations of the form (including the identity when $\left.S_{i}=I\right)$ :

$$
\left(V S_{i}\right)-(V)+(1-\theta) X_{i}=0
$$

The quantities $X_{i}$ being integral functions of certain roots of unity, and containing no numerical coefficients whose denominators are multiples of $p$, the equations obtained may be written

$$
\left(V S_{i}\right)_{p}-(V)_{p} \equiv 0 \quad(\bmod p) .
$$

From these it follows that the group $G$ considered has an invariant subgroup $H$ containing a subgroup of $K$ of order $p^{a-m+1}$, and theorem 14 is proved (cf. the arguments in the proof of theorem 10 in $L-G$ II).

\$4. There remains to prove that there is at least one non-vanishing determinant of $m^{2}$ elements in the matrix

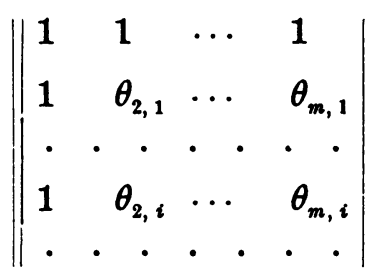

Since no two of the columns are identical, there must be at least one root $\phi \neq 1$ in the $r$ th column, $r \neq 1$, and, therefore, also one $\phi^{p b}=\rho \neq 1, \rho^{p}=1, \rho$ being one of the multipliers of the substitution $S_{h} \theta_{1, h}^{-1}$, say. If we exhibit the abelian group $K$ in the form

$$
K \equiv A\left(1+S_{h}+S_{h}^{2}+\cdots+S_{h}^{p-1}\right)+B\left(1+S_{h}+S_{h}^{2}+\cdots+S_{h}^{p-1}\right)+\cdots,
$$


$A, B, \ldots$ being substitutions of $K$ not identical with powers of $S_{h}$, it becomes evident that the sum of all the roots in the column considered can be written so as to have $1+\rho+\rho^{2}+\cdots+\rho^{p-1}$ as a factor and is therefore zero. Accordingly, by adding all the rows of (3) together, we get a row of the form

$$
p^{a}, 0,0, \ldots, 0 \text {. }
$$

Moreover, it is clear that we can multiply the rows by such roots of unity that their sum is

$$
0, p^{n}, 0, \ldots, 0 \text {; }
$$

etc. There results a determinant whose value is $p^{m a}$, and theorem 14 is fully proved.

$\S 5$. It now follows that, if the order of a collineation group $G$ in $n$ variables is divisible by $p^{n+n_{p}}$, when $p$ is a prime, and $n_{p}$ denotes the highest power of $p$ that divides $n$ !, then will $G$ contain a self-conjugate subgroup $H$, or be itself such a group. For, a group of order $p^{k}$ can be written in monomial form (theorem 9, $L$ - $G$ II). The group of order $p^{n+n_{p}}$ contained in $G$ will accordingly have an abelian subgroup of order $p^{n}$ at least, which subgroup, when written as a linear homogeneous group, will be of order $p^{n+l}$, if it contains a group of similarity-substitutions of order $p^{l}$.

COROLlaRY. The factor of the order of a collineation group $G$ (not containing $H$ ), which is the product of primes each $\leqq n$, must divide $n !(2 \cdot 3 \cdots p)^{n-1}$, where $2,3, \cdots, p$ denote all the different primes $\leqq n$.

$\S 6$. For the purpose of lowering the limit of $p(p>n)$, we shall consider the case of a group $G$ containing a substitution $S$ of order $p$ and of variety $m$, but no substitution of order $p k(k>1)$ unless such a substitution is the product of one of order $p$ and a similarity substitution. Let $V$ be any substitution of $G$. In the series of weights,

$$
(V),(V S), \cdots,\left(V S^{p-1}\right),
$$

write $+1,0$ or -1 for every root of unity of an index prime to $p$, according to the scheme explained in $\S 3$ of $L-G$ I. We shall, however, leave unchanged the roots of index $p$. The resulting expressions will be indicated by $[V],[V S], \ldots$ Then every $\left[V S^{a}\right]$ will be an integer lying between $-n$ and $+n$, inclusive, if the order of $V S^{n}$ is prime to $p$; otherwise $\left[V S^{a}\right]$ will be the sum of $n$th roots of unity, ${ }^{*}$ the negative of such a sum, or 0 , depending on the value $(1,-1$ or 0$)$ alloted to the multipliers of the similarity substitution $\left\{V S^{a}\right\}^{p}$. Let $w$ denote the number of weights $\left[V S^{a}\right]$ which are

* No primitive group in $n$ variables can have a substitution of order $p^{2}$, if $p>n$. See Cor. 1, page 316, of $L-G$ II. 
sums or the negatives of sums of $n p$ th roots of unity, and let $m^{\prime}$ be the least number of different, primitive $p$ th roots contained in any one of these weights $\left(1 \leqq m^{\prime} \leqq n\right)$. Then we shall prove that $w \geqq p+1-m-m^{\prime}$.

Let $\theta$ be a primitive $p$ th root of unity, and let $\theta^{a_{1}}, \theta^{a_{2}}, \ldots, \theta^{a_{p-m}}$ be the $p-m$ roots whose reciprocals are not found among the multipliers of $S$. Then we have

$$
\sum_{, r=0}^{p-1}\left[V S^{r}\right] \theta^{a_{i} r}=0 \quad(i=1,2, \cdots, p-m) .
$$

When $\theta$ is considered as a variable, the left hand members will not necessarily vanish, but we will have identities in $\theta$ of the form (by KRONECKER's theorem; see $\S 3$ of $L-G$ I):

$$
\sum_{r=0}^{p-1}\left[V S^{r}\right] \theta^{a_{i} r} \equiv\left(1+\theta+\theta^{2}+\cdots+\theta^{p-1}\right) X_{i},
$$

the quantities $X_{i}$ being integral functions of $\theta$ with integral coefficients. Let us operate upon these identities in turn by $\vartheta, \vartheta^{2}, \vartheta^{3}, \ldots(\vartheta=\theta \partial / \partial \theta)$, and put 1 for $\theta$ after differentiation. If we indicate $\left\{\theta^{a}\left[V S^{r}\right]\right\}_{\theta=1}$ by $A_{r}^{a}$, we obtain the congruences $(\bmod p)$

$$
\begin{aligned}
& \sum A_{r}^{1}+\alpha_{i} \sum A_{r}^{0} r \equiv 0, \\
& \sum A_{r}^{2}+2 \alpha_{i} \sum A_{r}^{1} r+\alpha_{i}^{2} \sum A_{r}^{0} r^{2} \equiv 0, \\
& \cdot \cdot \cdot \cdot \cdot \cdot \cdot \cdot \cdot \cdot \cdot \cdot \cdot \\
& \sum A_{r}^{p-2}+\cdots+\alpha_{i}^{p-2} \sum A_{r}^{0} r^{p-2} \equiv .^{0} \quad(i=1,2, \cdots, p-m),
\end{aligned}
$$

from which we derive the following :

$$
\begin{aligned}
& \sum A_{r}^{1} \equiv 0, \sum A_{r}^{0} r \equiv 0 ; \sum A_{r}^{2} \equiv 0, \sum A_{r}^{1} r \equiv 0, \sum A_{r}^{0} r^{2} \equiv 0 ； \\
& \sum A_{r}^{p-m-1} \equiv 0, \sum A_{r}^{p-m-2} r \equiv 0, \cdots, \sum A_{r}^{0} r^{p-m-1} \equiv 0 \quad(\bmod p) ;
\end{aligned}
$$

the summation in each case extending over the $p$ values of $r$.

Suppose that $w<p+1-m-m^{\prime}$. Then is every $A_{r}^{a}=0(a>0)$, except for at most $p-m-m^{\prime}$ subscripts $r$. For these subscripts we have

$$
\sum A_{r}^{1} \equiv 0, \sum A_{r}^{1} r \equiv 0, \cdots, \sum A_{r}^{1} r^{p-m-2} \equiv 0,
$$

and therefore every $A_{r}^{1} \equiv 0$, if $p-m-1 \geqq p-m-m^{\prime}$. We also find that every $A_{r}^{2} \equiv 0$, if $p-m-2 \geqq p-m-m^{\prime}$, etc. Finally every $A_{r}^{n^{\prime}} \equiv 0$.

Now, one of the quantities $\left[V S^{r}\right]$ had just $m^{\prime}$ distinct primitive $p$ th roots of unity, so that it may be written

$\left[V S^{r}\right]= \pm \alpha \pm \beta \theta_{1} \pm \gamma \theta_{2}+\cdots \pm \kappa \theta_{m^{\prime}} \quad(a, \beta, \cdots$ being positive integers $<n<p)$.

But, from $A_{r}^{1} \equiv 0, A_{r}^{2} \equiv 0, \cdots, A_{r}^{n^{\prime}} \equiv 0$ follow $\beta \equiv 0, \gamma \equiv 0, \cdots$, and 
therefore $\beta=\gamma=\cdots=0$, an absurdity. Hence the proposition :

$$
w \geqq p+1-m-m^{\prime} .
$$

Now, according to $\S \S 4-5$ of $L-G$ I, we have the congruence

$$
A_{r}^{0} \equiv a r^{m-1}+b r^{m-2}+\cdots=f(r) \quad(\bmod p),
$$

where $a, b, \ldots$ are certain integers. The numbers $A_{r}^{0}$ are integers all lying between $-n$ and $+n$ inclusive, and, by the proposition just proved, at least $p+1-m-m^{\prime}$ of them are either $+n$ or $-n$. However, at most $2(m-1)$ of the remainders of $f(r)(\bmod p)$ can be $+n$ or $-n$, unless $f(r)$ is merely a constant. This it could not be in all cases, as then, for every $V$ of $G$, we would have $(V)_{p} \equiv(V S)_{p}(\bmod p)$, and $G$ would have an invariant subgroup $H$, which would be of order $p^{l}$ and would therefore be abelian. In such a case $G$ could not be primitive (theorems II and III, $L-G$ I). Thus,

$$
2(m-1) \geqq p+1-m-m^{\prime} \quad \text { or } \quad p \leqq 3 m+m^{\prime}-3 \leqq 3 m+n-3 .
$$

We have now proved

Theorem 15. If a primitive collineation-group $G$ in $n$ variables has a substitution of order $p(p>n)$ and of variety $m(m \leqq n)$, but none of order $p k$, then is $p \leqq 3 m+n-3$.

If, in such a group, there is a substitution of order $p k$, which is the product of one $(S)$ of order $p$ and one of order $k$, then can the variety of $S$ be $n-1$ at most, unless there is an invariant subgroup $H$ (theorem 11, $L-G \mathrm{II}$ ). The number $p$ can then not exceed $(n-2)(2 n+1)$.

§7. If $n=4$, the theorem VI of $L-G$ I states that $p \leqq 13$. For $p=13$ we can, however, find no function $a r^{3}+b r^{2}+c r+d \neq d$, all of whose remainders $(\bmod 13)$ lie between -4 and +4 inclusive, and $13+1-8=6$ of which have the values +4 or -4 . Accordingly, by what precedes, a primitive group in 4 variables can have no substitution $S$ of order 13 unless it has one of order $13 k$, expressible as a product $S R\left(S^{13}=1, R^{k}=1 ; R\right.$ and $S$ permutable; $R$ not a similarity-substitution), whose weight is $\alpha_{1} \beta_{1}+\alpha_{2} \beta_{2}+\alpha_{3} \beta_{3}+\alpha_{4} \beta_{4}$, where $\alpha_{i}^{13}=1, \beta_{i}^{k}=1$. In this case we form the determinant corresponding to (3) of $L-G$ I, with $(T),\left(R^{a} S T\right),\left(R^{b} S^{2} T\right),\left(R^{c} S^{3} T\right),\left(R^{a} S^{r} T\right)$ for the elements of the first column. We will choose $a, b, c$ such that the resulting equation can be solved for $\left(R^{s} S^{r} T\right)$, and then replace all the 13th roots of unity by 1 . Now, a primitive group $G$ in 4 variables could not have an invariant subgroup $H$ of such a nature that, if $V$ and $S$ be any substitutions of $G$ and $H$ respectively, $(V)_{13} \equiv(V S)_{13}(\bmod 13)$. It follows that $\left(R^{s} S^{r} T\right)$ is not expressible in the form $A r+B$ ( $A$ and $B$ being independent of $r$ ), since in that event there would be just such a subgroup $H$. Excluding, therefore, 
this possibility, we find readily that three of the roots $\beta_{i}$ are equal to each other, say $\beta_{2}=\beta_{3}=\beta_{4}$, and then that

$$
\left(R^{s} S^{r} T\right)_{13} \equiv \beta_{1}^{s} A+\beta_{2}^{s}\left(B r^{2}+C r+D\right)(\bmod 13),
$$

the quantities $A, B, C, D$ being independent of $r$ and $s$.

If $A \equiv 0$, then is $\left(R^{s} S^{r} T\right)_{13} \equiv\left(S^{r} T\right)_{13} \beta_{2}^{s}(\bmod 13)$; or, putting $T=S^{-r}$, $\left(R^{s}\right)_{13} \equiv 4 \beta_{2}^{s}(\bmod 13)$. But this is an impossible congruence for $s=1$. Hence, $A \neq 0$, and we have

$$
\left(R^{s} S^{r} T\right)_{13} \beta_{2}^{-s} \equiv\left(\frac{\beta_{1}}{\beta_{2}}\right)^{s} A+B r^{2}+C r+D(\bmod 13) .
$$

The values $-1,0,1$ may now be assigned to the roots of unity involved, in accordance with the scheme of $\S 3, L-G \mathrm{I}$; and, as $\beta_{1} \neq \beta_{2}$, we have a function

$$
B_{1} r^{2}+C_{1} r+D_{1}+A^{\prime} \neq D_{1}+A^{\prime}(\bmod 13),
$$

$B_{1}, C_{1}, D_{1}$ and $A^{\prime}$ being integers, the last of which is capable of taking, at our will, at least two different values. All the remainders $(\bmod 13)$ of this function should lie between -4 and +4 inclusive. But such a function does not exist.*

In a similar manner we may deal with the cases $n=5, p=17$ or $19 ; n=6$, $p=23$. $\dagger$ The results are expressed in the following

TheOREM 16. The primes which may divide the orders of the primitive collineation-groups in 4, 5 and 6 variables are, respectively, not greater thar 11,12 and 19.

\section{BERLIN,}

January, 1906.

* In the article written by the author on quaternary groups, Mathematisohe A n nalen, vol. 60 (1905), pp. 204-231, it is proved, in a different manuer, that the primitive collineationgroups in 4 variables can have no substitutions of order 11 or 13.

† The cases $n=6, p=17$ or 19 have not been examined by the author. It is very likely that we may, by the process given above, throw out at least 19. 